\title{
Analysis of Lipid Vesicle Populations from Microscopy Video Sequences
}

\author{
Jernej Zupanc, Student Member IEEE, Erhan Bas, Deniz Erdogmus, Member IEEE
}

\begin{abstract}
Conducting research on lipid vesicles is very convenient, since they provide a stable and controllable environment for in vitro observations. Their resemblance to biological cell membranes allows biologists to assess hazardous potential of nanoparticles by exposing the vesicles instead of live organisms. When considering behavior of vesicles during incubation with nanoparticles, majority of existing research focus on observing single vesicles only. Our approach provides an ability to observe thousands of lipid vesicles for more representative behavior estimation. We developed an efficient algorithm to transform video sequences acquired with video microscopy into quantitative data. This includes steps required to filter noise, use multiple frames for more precise content presentation, detection of regions of interest, and segmentation of circular and non-primitively shaped vesicles. Presented work is a crucial step towards the creation of an automated computer analysis for lipid vesicles behavior assessment.
\end{abstract}

Index Terms - lipid vesicles, microscopy video, image segmentation

\section{INTRODUCTION}

With the advances in nanotechnology and engineered nanoparticles research, techniques for assessing properties of novel nanoparticles are also in great demand. Besides the desired properties of nanoparticles which make them interesting, we also have to determine their hazardous potential to humans, animals and biological systems in general [1]. Recent studies suggest that nanoparticles can strongly interact and disrupt cell membranes [2]. Given the large number of diverse nanomaterials, systematic approaches are favored. Beside in vivo assays [3], [4], a popular approach to assessing cell membrane permeability is to use artificial instead of biological systems [5]. It appears that palmitoyloleoylphosphatidylcholine (POPC) lipid vesicles, which are simplified biological membranes, offer controllable conditions to study effects of xenobiotics and can easily be observed directly under light microscope. In these experiments, populations of lipid vesicles are exposed to the various substances and observed through the microscope. Most published results focus on single vesicles and their morphological changes whether they are observed by a biologist spending hours behind the microscope, or a computer algorithm analyzing the video [6], [7]. However, with this approach, only a minute sample of the vesicle population can be observed. There is no guarantee that the changes we might be interested in will manifest in that precise sample. In our previous work we introduced a novel approach for analyzing larger samples [8], [9]. Microscopy images were taken at smaller

J. Zupanc jernej.zupanc@fri.uni-lj.si is with the Faculty of Computer and Information Science, University of Ljubljana, Slovenia. E. Bas and D. Erdogmus are with the ECE Department at Northeastern University, Boston USA. magnification, which allowed us to capture more vesicles. A biologist then manually labeled the vesicles and computer algorithms were used to quantitatively evaluate the labeled images (eg. quantities, sizes, eccentricities of vesicles). This way we were able to capture approximately $15 \%$ of the vesicle population we were interested in, and determine some significant effects which could not be acquired through single vesicle inspection. Apart from the benefits, this approach also had a weak point, since it was very time consuming for biologists. They spent weeks manually labeling tens of thousands of vesicles and preparing the images for the computer aided analysis. However, to repeat this experiment frequently with various nanoparticles, two things had to be improved: the percentage of captured vesicles had to be increased and the time needed to segment had to be vastly reduced. We decided to incorporate videos instead of images to capture more vesicles in the same amount of time and to develop an image processing algorithm for automatic vesicles segmentation. This paper describes the steps required to prepare the video of the vesicles and transform it into a useful data set with segmented lipid vesicles, prepared for statistical evaluation of vesicles' shapes and sizes.

\section{Methods}

\section{A. Acquiring data}

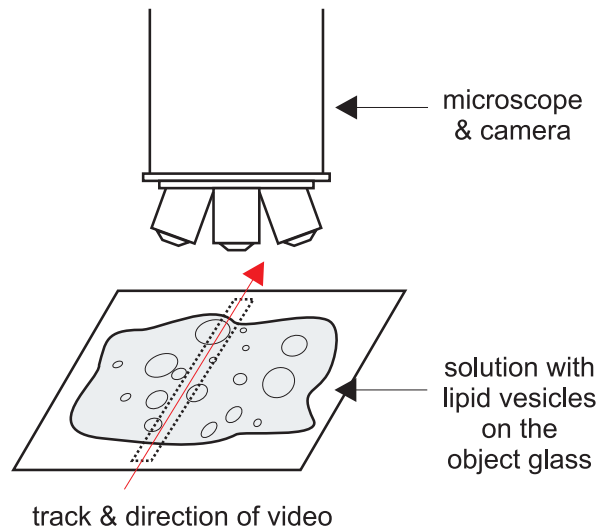

Fig. 1. Object glass is placed under the microscope. Videos are acquired by sliding the sample in one dimension which allows us to capture a single track.

The intact membrane of POPC lipid vesicles allows the sugar composition inside (saccharose) to differ from the composition outside (saccharose and glucose). This results in lipid vesicles appearing darker from the surrounding medium and thus easy for detection. The $45 \mu l$ of $5 \%$ glucose suspension containing lipid vesicles was applied on its own object 
glass and covered with a cover glass. The sample is observed under an invert microscope (Nikon Eclipse TE2000-S) with an attached camera at 400x magnification. This allows us to detect vesicles with radii larger than $1 \mu \mathrm{m}$. The field of view (visible area) at this magnification is $190 \times 143 \mu \mathrm{m}$. The population of lipid vesicles is contained on the object glass and spreads over a $15 \times 15 \mathrm{~mm}$ area. Each video was acquired by sliding the objective glass under the microscope in one dimension only (Fig. 1). Approximately 4 minutes are required to capture a single track at appropriate speed for the vesicles to remain detectable. Videos were uncompressed.

\section{B. Preprocessing}

The video segment used in the following analysis was a 33.467 second long sample at 15 frames per second (502 frames) and every frame of 768 x 576 pixels width and height. It was also cropped to eliminate a thin black border that contained no information. Despite the fact that both camera and microscope lenses were cleaned before the experiment, some lens noise remained visible throughout the video as seen in the Fig. 2-a. However, because of the static nature of the lens noise, we were able to remove it by calculating the median value of intensity over all frames and then subtracting its effect from each frame (Fig. 2-b). a

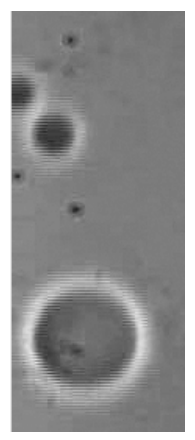

b

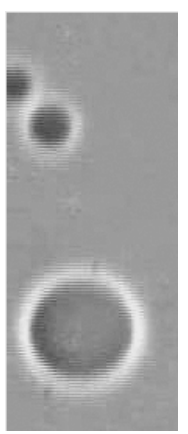

C

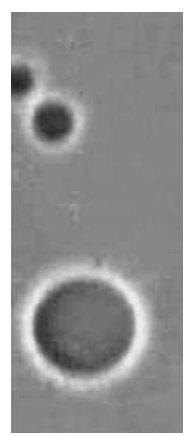

Fig. 2. a) Original. b) Without the lens noise. c) After downsampling and interpolating.

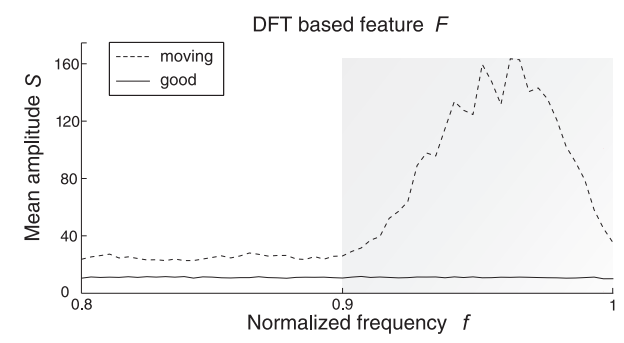

Fig. 3. Mean of the DFTs of the columns of a single frame. $F$ measure is calculated as the area under the mean curve within $10 \%$ high frequency band (shaded region).

While recording video, moving object glass under the microscope was performed manually, leading to the changing speed of movement. This resulted in frames corresponding to higher speed of movement being blurry. In order to use only the frames in focus for generating the panorama, the moving frames that were most distorted had to be identified. We manually labeled a minority of frames as good or moving and used Linear discriminant analysis (LDA) with two features to classify the rest of the frames. The first feature was the Brenner gradient, which can also be used as a blur metric [12]:

$$
B=\sum_{i=1}^{N} \sum_{j=1}^{M}[I(i, j)-I(i+m, j)]^{2}
$$

where $N$ and $M$ are height and width of the image, $m=2$, and $I$ is the intensity. The second feature we used $(F)$, is a Discrete Fourier Transform (DFT) based metric.

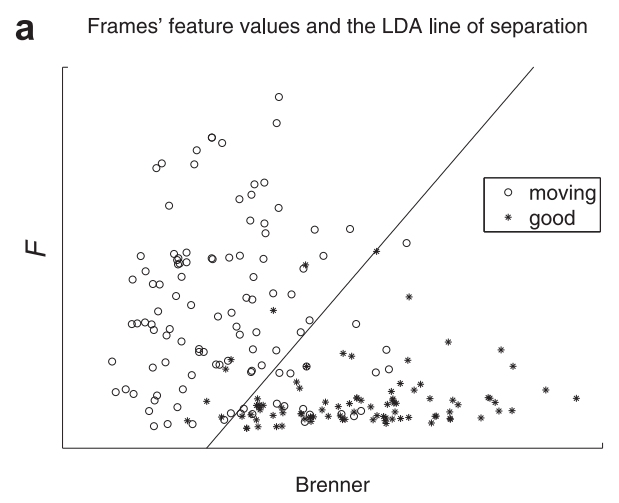

b Frames classified correctly with $>95 \%$ accuracy

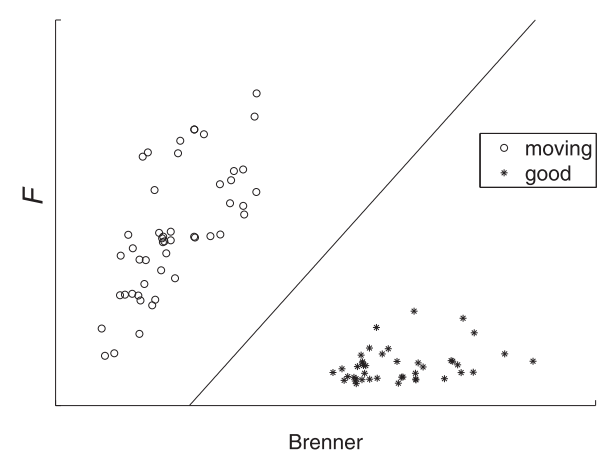

Fig. 4. a) Feature values of 200 test frames with the linear separation between classes. The line represents the LDA generated border between classes. b) Feature values of frames that have been classified with $>95 \%$ accuracy.

The reason for choosing the DFT was that we noticed horizontal interlacing artifacts (Fig. 2-a) in the moving frames, which suggested large amplitudes in higher frequencies for moving frames when compared to the good ones (Fig. 3). DFT based feature $F$ is given as the mean of the areas under the frequency curve in the high frequency region over the columns of a single image and can be written as $\sum_{f=0.9}^{1}\left(\sum_{i=1}^{M} S_{i}(f) / M\right)$, where $S_{i}(f)$ is the amplitude of the DFT for the $i^{t h}$ column. These two features were enough for the LDA to classify the frames as good or moving Fig. 4-a. We decided to remove only the frames which were classified as moving with more than 95\% accuracy Fig. 4-b. 


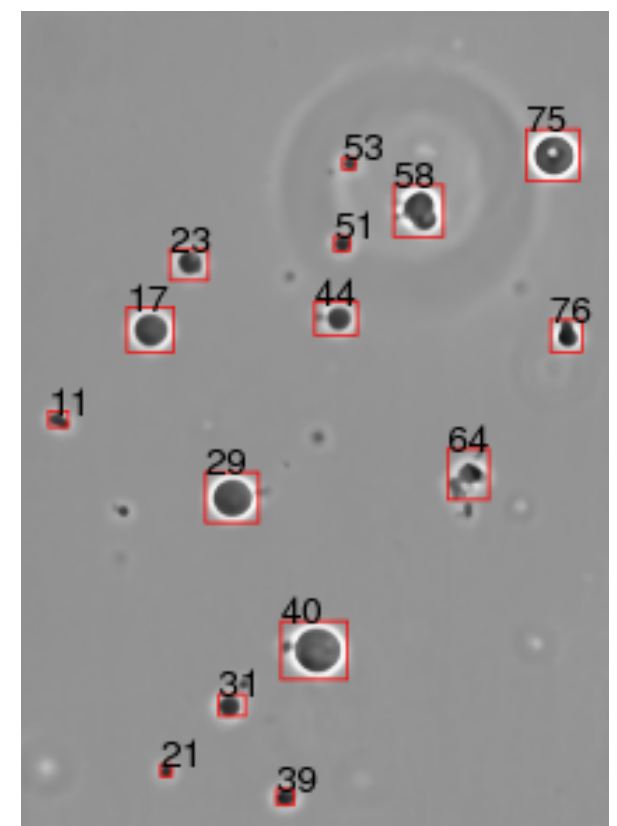

Fig. 5. Composed track with vesicles.

This allowed us to eliminate the blurred frames and limit the number of usable frames to 300 . Frames also had artifacts (horizontal lines) which were cleaned through vertical downsampling and interpolation Fig. 2-c. These frames were used to generate one big image containing the whole track covered in the video sequence.

Cross-correlation between frames was used to calculate the shift (only translation) of the camera movement. Frames were then aligned by change of correlation peaks position in consecutive frames. This resulted in a big image, where frames representing the same actual position in sample overlapped. To create the mosaic, median intensity of overlapping frames was calculated. In order to smooth the image while preserving the edges, we applied bilateral filter [10] with a 3 pixel spatial and 0.05 color domain kernel sizes, with a 5 x 5 window size.

\section{Defining and Segmenting within individual ROIs}

We implemented a foreground detection algorithm that uses the intensity and edge information. Sobel mask [11] is used to find the edges, and morphological closing operation is applied to extract the foreground regions. After hollow interior regions are filled with 4-connected neighbors, we calculate the bounding boxes (ROIs) encapsulating the detected foreground regions as shown in Fig. 5.

Since some ROIs contain more than one vesicle, further segmentation is required. A typical vesicle can be recognized as a dark region with a bright halo surrounding it. Fig. 6-a $\& \mathrm{~g}$ show the negative of a lipid vesicle and its surroundings with the detected b-box. In order to smooth the image and enhance vesicles, we employed bilateral filter (17 x 17 window size, 5 pixel spatial and 0.8 color domain kernel sizes) in combination with a Gaussian filter ( $7 \times 7$ window size with unit standard deviation) as presented in Fig. 6-b
$\&$ h. Next, we used intensity function of the filtered image to calculate the second order statistics. The Hessian defines a natural boundary for the vesicles. The region between the zero crossings is the high power portion of the signal we would like to segment. We approximate the derivative along $x \& y$-directions with differences. In 2D case, Hessian of the intensity, $I(x, y)$, at pixel location $(x, y)$ is given as

$$
H(x, y)=\left[\begin{array}{cc}
\frac{\partial^{2} I}{\partial x^{2}} & \frac{\partial^{2} I}{\partial x \partial y} \\
\frac{\partial^{2} I}{\partial y \partial x} & \frac{\partial^{2} I}{\partial y^{2}}
\end{array}\right] \rightarrow\left[\begin{array}{cc}
a & c \\
c & b
\end{array}\right]
$$

The second derivative test states that the eigenvalues of the Hessian are negative around local maximum. Both eigenvalues will be negative and their product is positive inside a region that is defined by the inflection curve of the intensity function. On the inflection curve, one of the eigenvalues will change the sign making the product negative. This can be detected by calculating the determinant of the Hessian which shares the sign with the product of eigenvalues of the Hessian. The determinant of each pixel location is calculated as $d=\left(a b-c^{2}\right)$, and the values are displayed in Fig. 6-c $\&$ i. Fig. 6-d \& j presents the regions where the determinant is positive. Inside the ROI, connected component analysis is employed to find and label the segments. Fig. 6-e \& $\mathrm{k}$ shows the result of the segmentation. However, signs of the eigenvalues by themselves do not provide sufficient knowledge to segment the vesicles. In order to eliminate the segments belonging to the background, we used the segmented regions together with the power of the intensity function. Power is given by the average of the squared intensity value of the negative image. Average power of the $i^{t h}$ segment can be given as

$$
P_{i}=\frac{1}{N_{i}} \sum_{x_{i}, y_{i} \in S_{i}} I\left(x_{i}, y_{i}\right)^{2}
$$

where $N_{i}$ is the total number of pixels, and $\left(x_{i}, y_{i}\right)$ are the pixel locations of the $i^{\text {th }}$ segment, $S_{i}$.

Let $P_{1}>P_{2}>\ldots>P_{k}$ be the average power of $k$ segments, and $d_{1} \ldots d_{k-1}$ the consecutive differences of the power values such that $d_{i}=P_{i+1}-P_{i}, i=1: k-1$. Number of vesicles, $j$, inside the ROI is given by

$$
j=\underset{i}{\operatorname{argmax}}\left(d_{i}\right) .
$$

An interesting yet difficult case is presented in Fig. 6bottom row, where tubular shaped vesicle is falsely detected as two separate regions. However, a small vesicle attached to the tubular shaped is correctly marked using the algorithm. In our future work, we will also focus on such non-primitively shaped vesicles. We tested the algorithm on the available video. Out of 92 regions our algorithm detected, $82 \%$ overlapped with the manually segmented ones. The remaining $18 \%$ include dust and other intruding particles which can be easily identified by biologists. As their manually labeled data was used as a gold standard for our current performance evaluation, we intend to incorporate their decision making in our future iterations of the algorithm. This could be done either by a semi-supervised segmentation or by incorporating a trained classifier. 


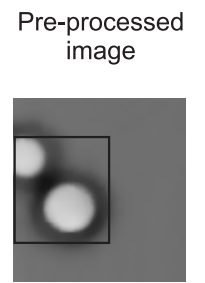

a

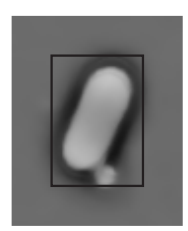

g

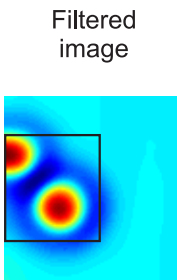

b

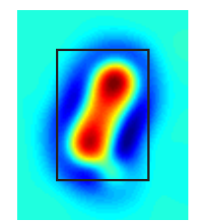

h

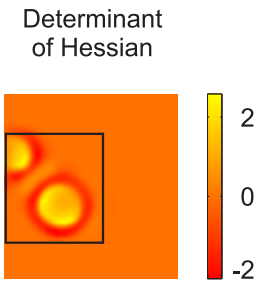

c

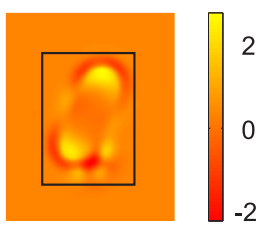

I
Determinant of Hessian $>0$

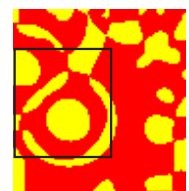

d

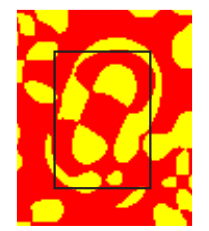

j
Segmented

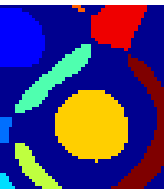

e

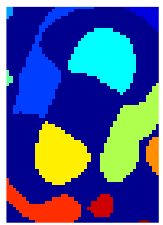

k
Result

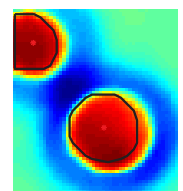

f

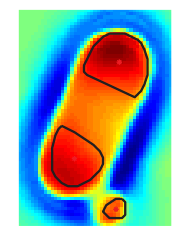

I

Fig. 6. Stages in segmentation process.

\section{Discussion}

The computerized procedure was designed to segment individuals in videos of populations of lipid vesicles. It is the first step towards a fully automated video analysis which is required for fast assessment of lipid vesicles behavior in various conditions. Video analysis research we conduct is synchronized with research of the team acquiring videos, and the observations each team presents are helpful for both. With creating and segmenting mosaics of video sequences, we were able to acquire $100 \%$ of the tracks in comparison with $15 \%$ acquired in the same amount of time by capturing single frames. Some of the problems we dealt with originate from the nature of the experiment and properties and state of the microscope and camera. For example, lens noise, frame interlacing, readjusting focus, variable speed of movement etc. Consequently, all of these problems had to be addressed and solved in the preprocessing steps in order to acquire image, good enough for accurate segmentation.

Currently the regions resulting from our segmentation can be used to count and estimate vesicles' sizes only. However, the biologists are also interested in detecting non-primitive shapes such as tubes, chains of vesicles, which occur more frequently when vesicles are exposed to membrane altering substances. In this respect, part of our future work will focus on extending the algorithm to classify all known shapes together with pointing out new, previously unidentified structures. Through the eyes of biologists, such advances would allow them to carry out more experiments in less time and therefore verify potential hazardous behavior or harmlessness of nanoparticles with greater certainty.

\section{ACKNOWLEDGMENTS}

The authors gratefully acknowledge the contribution of S. Boljte from Biotechnical Faculty of University of Ljubljana, Slovenia for acquisition of the data. Author Jernej Zupanc performed the work during visiting research at Northeastern University, sponsored by the Fulbright Scholarship.

\section{REFERENCES}

[1] N. R. Panyala, E. M. Pena-Mendez, J. Havel "Silver or silver nanoparticles: a hazardous threat to the environment and human health?" Journal of Applied Biomedicine, 6: pp. 117-119, (2008).

[2] P. R. Leroueil, S. Hong, et al. "Nanoparticle Interaction with Biological Membranes: Does Nanotechnology present a Janus Face?" Acc Chem Res., Vol. 40(5), pp. 335-342, (2008).

[3] J. Valant, D. Drobne, K. Sepcic, A. Jemec, et al. "Hazardous potential of manufactored nanoparticles identified by in vivo assay" Journal of Hazardous Materials, Vol. 171, 1-3, pp. 160-165, (2009).

[4] C. Zhen, M. Huan, et al. "Acute toxicological effects of copper nanoparticles in vivo", Toxicology letters, Vol. 163(2), pp. 109-120, (2006).

[5] R. Lipowsky "The conformation of membranes", Nature, Vol. 349, pp. 475-485, (1991).

[6] J. Pecreaux, H.G.Dobereiner, et al. "Refined contour analysis of giant unilamellar vesicles", Eur. Phys. J. E., Vol.13, pp. 277290, (2004).

[7] P. Peterlin, G. Jaklic, T. Pisanski 'Determining membrane permeability of giant phospholipid vesicles from a series of videomicroscopy images", Measurement Science and Technology, Vol. 20(5), pp. 055801055807,(2009).

[8] J. Zupanc, J. Valant, A. Dobnikar, et al. 'Interactions of Nanoparticles with Lipid Vesicles: A Population Based Computer Aided Image Analysis Approach", 31st Annual International Conference of the IEEE EMBS, pp. 1400-1403, (2009).

[9] J. Zupanc, J. Valant, V. Kralj Iglic, et al. "A new approach to analyze effects of nanoparticles on lipid vesicles", International Journal of Biomedical Nanoscience and Nanotechnology, Accepted.

[10] C. Tomasi, R. Manduchi, "Bilateral Filtering for Gray and Color Images" Proceedings of the Sixth International Conference on Computer Vision, pp. 839, (1998).

[11] R.C. Gonzalez, R.C. Woods, Digital Image Processing. AddisonWesley, Reading, MA, (1992).

[12] S. Yazdanfar, K. B. Kenny, K. Tasimi, et al. "Simple and robust image-based autofocusing for digital microscopy", Optics express., Vol 16(12), pp. 8670-8677, (2008). 